\title{
La tutela jurídica del patrimonio cultural bajo la influencia del soft law. Estudio del caso Misiones Jesuíticas Guaraníes en Argentina
}

\author{
The legal protection of cultural heritage under the influence \\ of soft law. Case study on the Guarani Jesuit Missions in Argentina
}

\section{Norma E. Levrand*}

\footnotetext{
* Abogada. Doctora en Derecho. Becaria posdoctoral de CONICET. Docente de la Universidad Nacional del Litoral y de la Universidad Autónoma de Entre Ríos, en Argentina.

$\bowtie$ normalevrand@gmail.com

http://orcid.org/0000-00021396-3688
}

RECIBIDO: [10.8.2018]

ACEPTADO: [3.10.2018]

\section{Resumen}

Este trabajo pretende identificar algunas modalidades específicas de influencia del soft law en el derecho interno argentino. Las normas de soft law constituyen regulaciones sin fuerza vinculante para los Estados. Son implementadas por el Poder Ejecutivo a través de la administración pública y varía el grado en el que son obedecidas. Mediante el estudio del caso de las Misiones Jesuíticas Guaraníes (inscritas en la Lista del Patrimonio Mundial de UNESCO) se reconocen y describen tres modalidades de intervención de normas de soft law en el ordenamiento interno argentino. El argumento central es que el proceso de incorporación, en la normativa nacional, de contenidos de soft law involucra agencias del nivel nacional, que, al bregar por esta incorporación, invaden competencias provinciales y municipales.

Palabras clave: patrimonio cultural, derecho internacional, instrumento internacional, acuerdo cultural, Argentina.

\section{Abstract}

This article aims to identify some specific modalities of soft law and their influence on the Argentinian domestic law. Soft law rules constitute regulations without 
binding force for the States. Their implementation is carried out by the Executive branch through the Public Administration, with a variable degree of obedience. By means of the case study of the Jesuit Missions of the Guaranis (inscribed in the UNESCO's World Heritage List) three modalities of intervention of soft law norms in the Argentinian internal order are recognized and described. The main argument is that the process of incorporating soft law content into national regulations involves agencies at the national level which, when struggling for this incorporation, invade provincial and municipal competences.

Keywords: cultural heritage, international law, international instruments, cultural agreements, Argentina.

Influir sobre una persona es transmitirle nuestra propia alma.

El retrato de Dorian Gray (1890), Oscar Wilde.

\section{Tabla de siglas y abreviaturas}

$\begin{array}{ll}\text { CNMLYBH } & \text { Comisión Nacional de Monumentos, de Lugares y Bienes Históricos } \\ \text { CONAPLU } & \text { Comisión Nacional Argentina de Cooperación con la UNESCO } \\ \text { ICOMOS } & \text { Consejo Internacional de Monumentos y Sitios } \\ \text { Mercosur } & \text { Mercado Común del Sur } \\ \text { OEA } & \text { Organización de Estados Americanos } \\ \text { UICN } & \text { Unión Internacional para la Conservación de la Naturaleza } \\ \text { UNESCO } & \text { Organización de las Naciones Unidas para la Educación, la Ciencia } \\ & \text { y la Cultura (sigla en inglés) }\end{array}$

\section{Introducción}

Uno de los efectos particulares de la globalización jurídica es la aparición de nuevas normatividades globales. A la regulación interna de los Estados-nación, que solo era exceptuada por tratados entre Naciones soberanas, se han agregado tratados multilaterales que crearon organismos supraestatales, convenciones universales que establecieron derechos unificados, y tribunales internacionales que sancionan el incumplimiento de tales normas. A la par de estas normas jurídicas aparecen otras, menos visibles pero igualmente profusas que recomiendan, reglamentan, insinúan ciertas líneas de acción para los Estados. 
Estas normatividades globales, denominadas soft law, surgen como alternativas a los tratados y la costumbre internacional, y han sido conceptualizadas como «expectativas de conductas pero que no son de aplicación obligatoria por los Estados» (Ratner, 1998, p. 65). Este tipo de regulación, que se ha incrementado exponencialmente, viene acompañada de la necesidad de implementación al interior del Estado (Galbraith y Zaring, 2014). Dicha implementación, habitualmente realizada por el Poder Ejecutivo, permite advertir que la administración es permeable al plano internacional.

La expresión soft law ha sido utilizada con diversos significados, entre los cuales la doctrina considera dos principales: por una parte se alude a instrumentos heterogéneos en los que se verifican dos elementos: el carácter jurídicamente no vinculante y una cierta relevancia jurídica; por otra parte se refiere al contenido de instrumentos, sean estos jurídicamente vinculantes o no (Mazuelos Bellido, 2004). Según Abi-Saab, la expresión nombra instrumentos cuya juridicidad (y fuerza vinculante) es ambigua o cuestionada (como se cita en Mazuelos Bellido, 2004). La particularidad de estas regulaciones es que no requieren una aprobación del Congreso, es decir que yacen dentro del dominio del Poder Ejecutivo (Galbraith y Zaring, 2014), lo que permite renegociarlas más fácilmente.

Los estudios acerca del funcionamiento de estas normas dan cuenta de su exuberancia en el ámbito del derecho comercial internacional (Hernández, 2018; Fillol y Márquez, 2018; Terpan, 2015; Martínez, 2017). También podemos encontrar algunos estudios de casos que visibilizan el impacto en la administración pública (Corvalan, 2018; Jurado, 2018; Calvo, 2018) y en el derecho ambiental (Foy Valencia, 2008). En estas investigaciones la doctrina ha advertido que la articulación entre ordenamientos jurídicos se ve conmovida por el proceso creciente de globalización en el cual las prácticas de múltiples agentes configuran procesos interrelacionados en los ámbitos nacional y global (Dezalay y Trubek, 1997). Pero consideramos que también puede visualizarse una penetración de normas internacionales en regulaciones y organismos subnacionales (provinciales y particularmente municipales). En estos casos, los organismos internacionales y sus agencias nacionales traducen estos lineamientos y procuran la operatividad de normas que pueden caracterizarse como soft law. En el ámbito de la tutela del patrimonio cultural, la consideración del modo en que influyen estas normas internacionales en el ordenamiento interno de los Estados ha sido escasamente problematizada hasta el momento (Levrand, 2016). Este trabajo pretende suscitar la reflexión acerca de esta cuestión a partir de un estudio de caso.

El análisis reflexivo del caso de las Misiones Jesuíticas Guaraníes (Argentina) permite inducir algunas modalidades efectivas de influencia del soft law en el derecho interno argentino. Argumentaremos que el proceso de incorporación, en la normativa nacional, de contenidos de soft law involucra agencias del nivel nacional, que, al bregar por esta incorporación, invaden competencias provinciales y municipales. 
La metodología de investigación utilizada ha sido cualitativa, a partir del análisis de un corpus documental complementado con entrevistas en profundidad a actores relevantes. El corpus documental se construyó principalmente (aunque no únicamente) a partir del archivo que posee la Comisión Nacional de Monumentos, de Lugares y Bienes Históricos (CNMLyBH en adelante). Asimismo, se utilizaron fuentes secundarias (esencialmente periódicos y bibliografía específica) para contextualizar algunas acciones desarrolladas por la CNMLyBH, como así también aquellas surgidas de las entrevistas.

El trabajo está estructurado en dos partes: la primera, de carácter general, introduce los conceptos esenciales referentes al ordenamiento jurídico del patrimonio cultural y las normas de soft law que comprende. Seguidamente presentamos de modo sucinto los antecedentes del caso a estudiar: las Misiones Jesuíticas Guaraníes en Argentina. La segunda parte del trabajo está dividida en tres apartados que analizan, respectivamente, tres situaciones en las cuales el soft law se involucra en el ordenamiento argentino y las consecuencias jurídicas de esta introducción. Por último, se presentan las reflexiones finales y la bibliografía citada.

\section{Los elementos constitutivos del caso: regulaciones, instituciones, actores, cosas}

\section{Aquellos elementos remotos: el alma de la regulación del patrimonio cultural}

A fin de comprender el contexto jurídico de producción del caso de estudio es necesario reconstruirlo. Para ello, consideraremos la Convención para la Protección del Patrimonio Mundial, Cultural y Natural como la norma jurídica central, en torno a la cual se congregan un conjunto de instituciones, actores y regulaciones a fin de propender a la tutela del patrimonio cultural.

La Convención para la Protección del Patrimonio Mundial, Cultural y Natural se aprobó en el seno de UNESCO en 1972 y fue ratificada por Argentina mediante la ley 21.836. Si bien este no es el primer instrumento internacional dedicado a la temática, su adopción implicó «el reconocimiento de la existencia de ciertos intereses comunes y superiores que sobrepasan los objetivos inmediatos y particulares de los Estados» (Blanc Altemir, 1992, p. 31).

La Convención estableció un régimen de tutela para aquellos bienes cuyo valor excepcional permite considerarlos parte del patrimonio común de la humanidad. Esta consideración se realiza «por afectación» al decir de A. Kiss (1982), a partir de su in- 
clusión en la Lista Representativa del Patrimonio Mundial, Cultural y Natural. Una vez que los bienes son incluidos en la lista, la comunidad internacional, carente de instituciones, confía a los Estados su protección en virtud del ejercicio de la competencia territorial que ejercen sobre estos (Silva, 1996). Es decir, que los Estados mantienen su soberanía sobre los bienes identificados como integrantes del patrimonio mundial.

La Convención asimismo crea el Comité del Patrimonio Mundial (unEsCo, 1972), organismo intergubernamental encargado, entre otras funciones, de establecer y mantener al día la Lista del Patrimonio Mundial y la Lista del Patrimonio Mundial en Peligro (art. 11 inc. 2, 4 y 5 de la Convención), de examinar el estado de conservación de los bienes inscritos en las listas a partir de los procesos de monitoreo reactivo y de informes periódicos (art. 11 inc. 7 y 29 de la Convención), y de definir los criterios que servirán para incluir bienes en cada una de las listas (art. 11 inc. 5 de la Convención).

Los criterios mencionados en el art. 11 inc. 5 de la Convención se han formalizado a través de las Directrices Prácticas para la aplicación de la Convención del Patrimonio Mundial, Cultural y Natural, cuya primera versión fue aprobada en junio de 1977 y desde entonces ha sido revisada más de una veintena de veces por el Comité (UNESCO, 2008). Las Directrices configuran el derecho al cual se sujeta el Comité como también los Estados parte que desean postular un sitio para su inclusión en las listas y también las oenegés que son organismos consultivos del Comité.

Además del Comité del Patrimonio Mundial, para el estudio del caso Misiones Jesuíticas Guaraníes resulta relevante considerar la actuación del Bureau del Patrimonio Mundial. Este es un organismo de apoyo administrativo que fue establecido por el Comité. Se reúne durante los períodos de sesiones del Comité con la frecuencia que se considere necesaria y es responsable de los asuntos cotidianos del Comité. Está compuesto por los representantes de siete Estados parte, elegidos anualmente por el Comité.

Si bien no es objeto de este trabajo explicar el proceso de postulación, evaluación e inclusión de un bien en la Lista del Patrimonio Mundial, consideramos relevante aclarar que la evaluación de la postulación realizada por los Estados parte está a cargo de oenegés internacionales que actúan como organismos consultivos del Comité. En el caso del patrimonio cultural, corresponde al Consejo Internacional de Monumentos y Sitios (ICOMOS) evaluar las postulaciones y aconsejar, o no, su inclusión en la lista (art. 13 inc. 7 de la Convención). IComos es una organización no gubernamental con sede en Francia, compuesta por expertos de diversas disciplinas, como la arquitectura, la arqueología, la historia, la historia del arte, la geografía, la antropología, la ingeniería y el urbanismo. Fundado en 1965, tiene como objetivo la aplicación de teorías y métodos científicos en la conservación del patrimonio arquitectónico y arqueológico, basando su trabajo en los principios de la Carta Internacional sobre la Conservación y la Restauración de Monumentos y Sitios (Carta de Venecia) de 1964. 
Ariño Villarroya anota como una paradoja de la construcción de un patrimonio de la humanidad el hecho de que la proyección a escala global produce «una pérdida de control local [...] un desarraigo y desterritorialización» (2002, p.146). En este sentido, un conjunto de actores globales, como ICOMOS, la UICN, la World Monuments Fund o el Banco Interamericano de Desarrollo establecen regulaciones de soft law que son acogidas por organismos nacionales.

En el ámbito del patrimonio cultural, cobran especial relevancia ciertos documentos, entre los que podemos mencionar las cartas y recomendaciones aprobadas en el seno de organismos y oenegés internacionales sin fuerza vinculante para los Estados, que procuran establecer principios y normas de conducta para la conservación de los sitios patrimoniales. A ellas se suman las Directrices Prácticas, consideradas un instrumento de soft law en tanto han sido aprobadas en el seno de un organismo intergubernamental (el Comité del Patrimonio Mundial) sin exigir que los Estados parte las ratifiquen. Configuran una reglamentación de la Convención del Patrimonio Mundial que, en algunos casos, ha extendido su vigencia hacia áreas no específicamente establecidas en la norma, tales como el entorno de los sitios del patrimonio mundial y nuevas categorías, como paisajes culturales e itinerarios culturales.

En el procedimiento de inclusión de un bien en la Lista del Patrimonio Mundial, ICOMOS, como organismo consultivo del Comité, produce una evaluación de los sitios. En esta establece el valor universal excepcional del bien y, en algunos casos, también realiza recomendaciones que no poseen carácter vinculante.

El ingreso de estas normas no vinculantes se produce, como indican Galbraith y Zaring (2014), a partir de su adopción por los organismos administrativos nacionales. En el caso argentino, cobra especial relevancia la actuación de la CNMLyBH. Esta comisión, creada por la ley 12.665 en 1940, ejerce la superintendencia sobre los monumentos, lugares y bienes históricos nacionales (art. 1 ter ley 12.665). En el caso estudiado, como en la mayoría de los bienes culturales argentinos incluidos en la Lista del Patrimonio Mundial (Levrand, 2017), estos bienes fueron reconocidos como monumentos nacionales antes de postularse ante UNESCO.

Conforme al texto legal indicado, la CNMLyBH interviene necesariamente ante cualquier circunstancia en la cual el bien sea susceptible de modificaciones en su estatus jurídico (venta, gravamen, enajenación, etc.) o en su materialidad (refacción, conservación, alteración, etc.). Ello implica que, en los bienes que conforman el caso estudiado, la CNMLyBH se instituye como un actor necesario en el proceso de postulación y en la gestión de los bienes. 


\section{Estos elementos próximos: los bienes y actores de un sitio del patrimonio mundial}

El caso de estudio de este trabajo corresponde a la postulación y posterior inscripción del sitio Misiones Jesuíticas Guaraníes, en Argentina. A pesar de la limitación geográfica de esta postulación, la obra evangelizadora de los sacerdotes jesuitas se extendió, a partir de 1609, en territorios que hoy corresponden a Brasil, Paraguay y Argentina. En este último país se situaron 15 misiones guaraníticas, 11 de las cuales se encontraban en la actual provincia de Misiones y 4 en lo que hoy conocemos como Corrientes. Tuvieron su apogeo durante el siglo XVIII. A partir de 1768, año de expulsión de América de la Compañía de Jesús, comienza la decadencia de estos poblados y su destrucción a raíz de la guerra emprendida contra los guaraníes.

La selva amazónica cubrió los restos de estas misiones hasta que la colonización proveniente de la inmigración europea, a fines del siglo XIX, exigió que las autoridades ubicaran y trazaran colonias agrícolas. En aquel momento se sugería que el centro del pueblo fueran las ruinas y la plaza, la jesuítica. De este modo, los restos podían ser reciclados para ser utilizados como edificios públicos o particulares, concibiéndose de este modo su rescate (L. Salvatelli, comunicación personal, 24 de agosto de 2016).

De las 15 misiones guaraníticas existentes en el territorio argentino, 4 fueron escogidas para la postulación como patrimonio mundial: San Ignacio Miní, Loreto, Santa Ana y Santa María la Mayor. Estas se ubican en la provincia de Misiones y han sido objeto de diversas intervenciones para su recuperación y puesta en valor (Onetto, 1999; Levrand, 2017). Los cuatro sitios han sido declarados Monumento Histórico Nacional y puestos bajo la superintendencia de la CNMLyBH.

La inclusión de las ruinas en la Lista del Patrimonio Mundial Cultural se produjo en 1984. Carlos Onetto (1999) indica que esta declaración surgió a partir de una visita del director general de la UNESCO a Argentina en 1981. En esa oportunidad recorrió las ruinas jesuíticas existentes en Misiones, y convocó a Argentina, Brasil y Paraguay a una reunión tripartita a fin de concertar sobre la preservación de las misiones jesuíticas de guaraníes. La reunión se llevó a cabo en noviembre del mismo año, y estipularon entonces medidas generales y de orden particular para ciertas ruinas, como también medidas de protección.

En 1982 fue designado vicepresidente del Bureau del Comité del Patrimonio Mundial el profesor Carlos Gelly y Obes, quien por entonces ocupaba interinamente el cargo de presidente de la CNMLyBH. Como tal, recomendó la inclusión en la Lista del Patrimonio Mundial de la misión de San Miguel del Brasil, e hizo constar que ese bien era integrante de la serie monumental jesuítica americana —art. 1 inciso segundo de la Convención del Patrimonio Mundial, Cultural y Natural- (Gelly y Obes, 1983). La 
serie se completaría con la presentación que iniciaría Argentina con las ruinas de San Ignacio Miní y Santa María la Mayor (UNESCO, 1983). Esta intervención fue referenciada, luego, por integrantes de la CNMLyBH como definitoria para que se incluyeran las ruinas en la Lista del Patrimonio Mundial.

Al examinar los documentos, verificamos que al evaluar la postulación de la Misión de San Miguel realizada por Brasil, ICOMOS recomendó inscribir tal sitio en el marco de una nominación global concerniente a las misiones jesuíticas de América Latina que asociara la proposición de varios Estados miembro. Posteriormente, al evaluar la presentación argentina (20 de septiembre de 1983), ICOMOS indica que, en la sesión de 1983 del Comité del Patrimonio Mundial, Argentina anunció oficialmente la proposición de inscripción de cuatro reducciones guaraníes de las quince que se encuentran en su territorio. Esta elección se justificó en el interés histórico y arqueológico como así también en cierta complementariedad entre estas. En virtud de ello, y del criterio de proposición global de las misiones jesuíticas definido en 1983 por el Comité del Patrimonio Mundial, ICOMOS recomienda la inscripción de los cuatro bienes sometidos a examen (UNESCO, 1984). En la octava sesión del Comité del Patrimonio Mundial, se incluyeron en la Lista las cuatro misiones nominadas por Argentina en virtud del criterio iv) de evaluación del Valor Universal Excepcional: «ser un ejemplo eminentemente representativo de un tipo de construcción o de conjunto arquitectónico o tecnológico, o de paisaje que ilustre uno o varios periodos significativos de la historia humana» (UNESCO, 2008, par. 77).

Como exponemos en la segunda parte de este trabajo, tanto en el proceso de postulación como posteriormente, en la gestión del sitio Misiones Jesuíticas Guaraníes las normas de soft law tuvieron notable injerencia en el gobierno del territorio. La adopción de estas regulaciones por parte de la administración nacional tiene particulares efectos en Argentina, cuya estructura de gobierno es federal.

La estructura federal argentina se realiza a partir de tres principios: la supremacía del orden jurídico federal, la participación de las provincias en la formación de la voluntad federal y la distribución de competencias al interior del nivel nacional (Bidegain, 2001). Esta última puede considerarse a partir de la distribución material o funcional. La Constitución Nacional establece el principio de la competencia material en el artículo 121, acordando a las provincias todo el poder no delegado. No obstante, la flexibilidad de algunas normas constitucionales permite una actuación del gobierno federal más extensa que la definida anteriormente. Así, en el ámbito del patrimonio cultural, las facultades de reglamentar el comercio internacional e interprovincial (Constitución Nacional, art. 75 inc. 13) y las cláusulas de prosperidad y desarrollo (Constitución Nacional, art. 75 incs. 18 y 19) proporcionan sustento jurídico al afán de regulación nacional de la temática. De esta manera, la textura abierta de muchas normas constitucionales unida a una dinámica del federalismo que se ha modificado a 
partir de la centralización de los poderes delegados y del cambio de la infraestructura socioeconómica (Frías, 2011) demandan el empeño de directivas de interpretación acordes a cada caso concreto.

A ello se suma la distribución competencial entre provincias y municipios, afirmada a partir del reconocimiento constitucional de la autonomía municipal y delimitada tanto en constituciones provinciales como en leyes orgánicas municipales. Esta distribución encierra las mismas problemáticas de interpretación que el nivel nacional, y tolera igualmente la influencia del soft law en la gestión a su cargo.

\section{Influencia de las normas de soft law en la postulación y gestión de las Misiones Jesuíticas Guaraníes}

\section{El soft law como motor del proceso político}

Para dar cuenta de la primera modalidad de influencia del soft law en el ordenamiento interno consideraremos la evaluación de IComos del sitio San Miguel de las Misiones (Brasil), a la que referimos en el apartado anterior. En virtud de ella, se recomendaba la inscripción del sitio en el marco de una propuesta global que comprendiese las principales misiones jesuíticas de América Latina. En el ámbito de la Convención del Patrimonio Mundial, Cultural y Natural, la principal función de ICOMOS es «evaluar los bienes propuestos para ser incluidos en la Lista del Patrimonio Mundial» (UNESCO, 2008, par. 35) y esta evaluación es orientativa para el Comité (UNESCO, 2008, par. 151). De hecho, aunque el Comité no está vinculado por las evaluaciones y recomendaciones de ICOMOS, ${ }^{1}$ en general ellas son seguidas y el Comité no utiliza su capacidad de desviar su decisión (Zacharias, 2010).

Cuando esta evaluación fue puesta a consideración del Bureau del Comité del Patrimonio Mundial, el representante de Argentina (que ocupaba la vicepresidencia del Bureau) se comprometió a presentar las misiones de San Ignacio Miní y Santa María la Mayor. ${ }^{2}$ Esto determinó que la declaración de inscripción de San Miguel de las Misiones se hiciese en la categoría conjuntos en virtud del compromiso de presentación argentino. Esta categoría refiere a aquel «grupo de construcciones, aisladas o reunidas,

Esta es la interpretación del propio comité, realizada en la 20.a sesión, 1996, en la cual se indicó que no era una práctica corriente seguir las decisiones del Bureau o de los organismos consultivos como si fuesen vinculantes (UNESCO, 1997).

2 En aquel momento (1983), Paraguay no había ratificado aún la Convención del Patrimonio Mundial, por lo que el único país en la región con posibilidades de presentar sitios de estas características para su inscripción era Argentina. 
cuya arquitectura, unidad e integración en el paisaje les dé un valor universal excepcional desde el punto de vista de la historia, del arte o de la ciencia» (UNESCO, 1972).

En este caso, la evaluación que realizó ICOMOS aceleró y comprometió la presentación argentina. El importante rol que juega esta oenegé internacional en la legitimación de la protección internacional del patrimonio cultural ha sido referenciada como una debilidad de la Convención por algunos autores (Zacharias, 2010; Affolder, 2007; Muriel, 2010). Por una parte, la conformación científica de este organismo consultivo refuerza las decisiones del Comité del Patrimonio Mundial al ofrecerlas de modo más objetivo y menos político, favoreciendo su cumplimiento. Por otra parte, sin embargo, cierra la posibilidad de participación democrática legítima, atento a que se requiere una gran formación para cuestionar estos dictámenes. Indica S. Maswood que esta participación de los organismos consultivos ha sido efectiva para "persuadir a los Estados parte a revisar y rescindir las decisiones que de otro modo podrían dañar los sitios importantes del patrimonio» (como se cita en Affolder, 2007, p. 64).

Así, aunque la evaluación realizada para el sitio propuesto por Brasil no tenía fuerza vinculante ni, en principio, para el Comité del Patrimonio Mundial ni para el Estado Argentino, desencadenó una decisión política que tradujo una norma de soft law: la decisión 07com VIII del Comité (UNESCo, 1984). Así se inscribió en la Lista del Patrimonio Mundial el sitio de San Miguel de las Misiones (Brasil), considerando que formaba parte de un conjunto que se completaría con la postulación de los dos sitios argentinos.

Las decisiones del Comité del Patrimonio Mundial son decisiones de casos individuales, por ende, pueden equipararse a decisiones ejecutivas desde el punto de vista del derecho nacional. Es decir que, en principio, esta decisión no obligaba al Estado argentino, quien no había realizado la postulación.

No obstante, una interpretación de la integridad de la Convención del Patrimonio Mundial, Cultural y Natural (UNESCO, 1972) da cuenta de que establece un mecanismo de gobierno de este tipo de bienes que se basa en una acción cooperativa de los Estados. En este sentido, reconoce que la comunidad internacional tiene el deber de prestar asistencia técnica, económica u otra a los Estados parte para que estos puedan proteger y conservar el Patrimonio Mundial que se encuentra en sus territorios (art. 6 inc. 1). El sentido que trasciende de su articulado es que los Estados parte asumen el compromiso de proteger este patrimonio universal. Este mecanismo cooperativo se complementa con un órgano que posee la atribución de determinar o incluso crear deberes que incumben a los Estados parte, nos referimos al Comité del Patrimonio Mundial.

En ningún caso un bien puede inscribirse en esta Lista sin el consentimiento del Estado en el cual se encuentra. Solo el gobierno del país en el que se ubica el sitio puede postularlo para la inscripción y a este fin debe demostrar un compromiso que se 
manifiesta en el reconocimiento de la legislación protectoria pertinente, la dotación de personal y la existencia de planes de manejo y de financiamiento del bien (Gerbert, 1998).

En este caso, más bien, nos encontramos ante un compromiso político del Estado argentino. Como tal, no es vinculante jurídicamente. Pero dada su naturaleza, era factible que se cumpliese, y así, en septiembre de 1983 fue presentada la documentación sobre San Ignacio Miní. Para lograrlo, el engranaje administrativo al interior de Argentina (fundamentalmente la CNMLyBH, pero también la Dirección de Tierras del Ministerio de Asuntos Agrarios de la provincia de Misiones) ${ }^{3}$ se puso en funcionamiento. No solo se produjeron investigaciones e informes sino que se modificaron normas jurídicas (como la titularidad dominial de los terrenos colindantes a las ruinas, mediante decreto provincial no 2019/1982).

\section{El soft law como promotor de normativa nacional y subnacional}

La segunda modalidad de influencia del soft law proviene de la regulación del entorno del sitio declarado Patrimonio Mundial Cultural. Esta consideración del entorno, originada en documentos internacionales de soft law, provenientes de organizaciones no gubernamentales (como ICOMOS) y organizaciones internacionales (como OEA), ${ }^{4}$ fue incluida en las Directrices bajo la denominación «protección de la zona de amortiguamiento» (UNESCO, 2008). Si bien al momento de la inscripción de las Misiones Jesuíticas Guaraníes en la Lista del Patrimonio Mundial el establecimiento de esta zona de amortiguamiento no era obligatoria, el Comité del Patrimonio Mundial realizó una advertencia al Estado argentino indicando la necesidad de proteger el entorno de las misiones (UNESCO, 1984).

En 1998, la Comisión Técnica del Convenio Nación-Provincia ${ }^{5}$ comenzó a tratar la problemática suscitada por el emplazamiento de un complejo industrial yerbatero en

3 A fin de incorporar al monumento las tierras fiscales lindantes, el gobernador de facto de la provincia ordenó, en 1982, al ministro de Asuntos Agrarios que, a través de la Dirección de Tierras, determinase los instrumentos legales para su pasaje a dominio de la CNMLyBH, e incluyera la «erradicación de intrusos» (Schávelzon, 2008, p. 404).

4 Entre ellos cobran especial relevancia la Recomendación relativa a la Protección de la Belleza y del Carácter de los Lugares y Paisajes (UNESCO, 1963); las Normas de Quito (1967), que proponen un nuevo dimensionamiento de la espacialidad de los monumentos, y la Carta de Venecia (1964), que plantea la tutela de «la creación arquitectónica aislada, así como también el sitio urbano o rural...» (ICOMOS, s. f.).

5 La gestión del sitio Misiones Jesuíticas Guaraníes se presentó compleja desde el inicio del proceso de postulación, atento a que algunas ruinas eran del dominio nacional, en tanto que otras pertenecían a la provincia de Misiones e incluso ciertos sectores habían sido enajenados a particulares. Por ello, a partir de la década de 1970 se sucedieron una serie de convenios entre la Nación y la provincia de Misio- 
las inmediaciones de la Misión de Santa Ana. Este municipio contaba con una protección del entorno de la Misión, ${ }^{6}$ y había aprobado la instalación del complejo industrial fuera de la zona protegida. Algunos actores entendieron que, a pesar de estar fuera del entorno de la Misión, este complejo afectaba el contexto ambiental del sitio (J. Pozzobon, comunicación personal, 18 de agosto de 2016). En este sentido, la arqueóloga Lorena Salvatelli explicó:

[...] en los alrededores de las reducciones muchas veces hay capillas interreduccionales o puestos de estancia [...] porque la gente llevaba productos de una reducción a otra, se movilizaba [...] entonces estas capillas formaban parte del sistema de descanso y de oración entre reducción y reducción (L. Salvatelli, comunicación personal, 24 de agosto de 2016).

Los vestigios de estas postas del sistema reduccional fueron relevados por la arqueóloga Ruth Poujade, pero se han perdido a raíz de las intervenciones posteriores en el sitio.

Por su parte, el municipio alegaba la creación de puestos de trabajo, que revitalizarían una economía que comenzaba a dar muestras de la crisis (J. Pozzobon, comunicación personal, 18 de agosto de 2016).

Este conflicto «aceleró la necesidad de desarrollar un proyecto de redefinición del acceso al Conjunto Reduccional de Santa Ana», y conforme la Comisión Técnica logró un acuerdo para modificar el acceso «con la finalidad de morigerar el impacto que tiene el actual acceso y generando con ello también un valor adicional, tal lo constituye la reconstitución del ingreso histórico al conjunto reduccional» (Comisión Técnica del Convenio Nación-Provincia, 1998).

También la CNMLyBH había expresado su preocupación ante la Comisión Nacional Argentina de Cooperación con la UNESCo (CONAPLU), en febrero de 1999, resaltando la contravención que la instalación del complejo producía a cartas internacionales de conservación del patrimonio «cuyos postulados, a los que esta Comisión adhiere, sostienen que el entorno ambiental de un monumento debe ser preservado porque integra con este un todo indisoluble» (CNMLyBH, 1999).

La situación llegó a conocimiento de UNESCO, quien en febrero de 1999 solicitó información acerca del emprendimiento, como así también de las medidas adoptadas o previstas por el Estado que garanticen la preservación del monumento y su entorno

nes a fin de concertar las acciones en relación con este bien cultural. Estos convenios establecían comisiones ejecutivas y técnicas.

6 La ordenanza municipal 8/92, del 15/09/92, estableció una zona de no innovar en las inmediaciones del Complejo Jesuítico de Santa Ana, con lo que prohibió cualquier acción que implicara una alteración en el equilibro entonces existente que no fuera aprobada por el área cultural del gobierno provincial y municipal. 
(nota WXC/74/201/041/HvH/cc, 1999). La respuesta del Estado argentino se fundamentó en los siguientes argumentos centrales: $a$ ) el complejo industrial se halla fuera del entorno jurisdiccional del conjunto jesuítico de Santa Ana, a 700 metros de la Plaza Central de la Reducción; $b$ ) entre el conjunto reduccional y el complejo industrial ha sido declarada una zona de protección y de no innovar por el Municipio de Santa Ana; c) la infraestructura industrial no se halla asentada sobre restos arqueológicos y no afecta visual ni paisajísticamente la jurisdicción del monumento; $d$ ) se ha proyectado una reubicación del ingreso reconstituyendo el trazado histórico que vinculaba las reducciones (Secretaría de Cultura, 2000).

Finalmente, en su 23. a sesión, el Bureau del Patrimonio Mundial concluyó que la planta industrial no tenía impacto visual sobre el Patrimonio Mundial Cultural y tomó nota del nuevo acceso que se construiría (Centro del Patrimonio Mundial, 2000). Asimismo, siguiendo una recomendación de ICOMOS, el Bureau requirió que las autoridades argentinas definieran zonas de amortiguamiento en las Misiones Jesuíticas e informaran a la Secretaría las medidas tomadas al efecto.

Sin embargo, la cnмlyвн solicitó al Municipio de Santa Ana, en diciembre de 2000, que retrotraiga «a su estado original la zona de amortiguación de Santa Ana» en virtud del compromiso asumido por el país por la Convención del Patrimonio Mundial. En caso de que no fuera posible erradicar la planta industrial, que disponga el cambio de acceso al conjunto, de forma tal que los galpones queden fuera del circuito turístico (сNмLyвн, 2000). La respuesta del intendente municipal no se hizo esperar y el 15 de enero de 2001, previa enumeración de las acciones llevadas a cabo por el municipio (declaración de zona de no innovar, cambio de acceso) solicitó un subsidio para hacer frente al cambio de acceso propuesto y aceptado. No obstante, por falta de recursos económicos, este acceso nunca pudo efectivizarse (J. Pozzobon, comunicación personal, 18 de agosto de 2016).

Como puede observarse, el soft law aplicable al caso operó como una fuerza que impulsó el desarrollo de principios y criterios, y marcó el derrotero de la normativa nacional. Así, la ordenanza municipal 08/92 fundamenta, en sus considerandos, que el monumento cobra sentido en virtud de su contexto ecológico, con el cual está imprescindiblemente integrado (obsérvese la similitud de la expresión con aquella derivada de la Carta de Venecia (ICOMOS, s. f.) «El monumento es inseparable [...] del lugar en el que está ubicado» o de las Normas de Quito (1967) «La idea de espacio es inseparable del concepto de monumento».

Al mismo tiempo, este caso plantea una cuestión de competencia legislativa que debe ser analizada a la luz del federalismo argentino. Por una parte, el Estado nacional se obligó, en virtud de la ratificación de la Convención del Patrimonio Mundial, a garantizar el cumplimiento de las pautas establecidas por unESCO para la salvaguarda de los bienes inscritos en la Lista del Patrimonio Mundial (arts. 4, 5 y 6.2 de la Conven- 
ción, UNESCO, 1973). Entre estas pautas, derivada de un documento de soft law, se encuentra la protección del entorno del bien. Evidentemente ello exige una normativa específica que en casos - como el estudiado- en los cuales el bien se encuentra bajo el ejido municipal será competencia del municipio. Para González Bertomeu, se trata de un típico problema de agencia, en el que el responsable a nivel internacional por el cumplimiento de la Convención es el Estado nacional, a pesar de que las violaciones pueden provenir de las provincias o los municipios, que no son técnicamente parte en él (2010).

Algunas posibles soluciones se han dado en referencia a la aplicación de tratados de derechos humanos (Dulitzky, 2006). Descontando que en esos casos no nos encontramos ante soft law, sino ante normas jurídicamente vinculantes y que implican responsabilidad internacional, mencionaremos las dos soluciones propuestas a fin de analizar su procedencia en el caso examinado. Para algunos autores, el Congreso Nacional está autorizado a crear leyes con estándares mínimos sobre interpretación y aplicación de estos derechos consagrados por tratados internacionales, lo que implicaría una transferencia de poder de las provincias a la nación. Otros entienden que la solución puede establecerse en virtud del diseño institucional, que impone a la Corte Suprema la custodia de los derechos constitucionales, con lo que permitiría la fijación de estándares mínimos en esta materia.

Ninguna de ambas propuestas satisface los particulares requerimientos de la aplicación, en el nivel subnacional, de una norma internacional de soft law. Su carácter no vinculante exime al Estado nacional de la responsabilidad internacional, por lo cual no fundamenta adecuadamente una delegación de competencia desde los municipios hacia el Congreso Nacional. Por otra parte, la fijación de estándares judiciales exigiría recurrir a este cuerpo del Estado. La problemática de la inserción de la regulación del entorno ha generado cuestionamientos en más de un sitio (Levrand, 2016), a pesar de lo que la situación en ningún caso ha llegado a conocimiento de la Justicia. En cada uno de los casos estudiados la solución se ha obtenido por diversos medios, que incluyen acuerdos políticos o que se desactivara el conflicto al asumir nuevas autoridades políticas y se desinteresaran de la problemática. Este es el caso de las ruinas de Santa Ana, en las cuales, más allá de la ordenanza dictada, no han ocurrido innovaciones desde el año 2001.

\section{El soft law como instrumento que interfiere en la dialéctica del Poder Ejecutivo y Poder Legislativo}

La tercera modalidad de influencia del soft law en el derecho nacional que analizamos se refiere al pedido de informes que realizó la Cámara de Senadores al Poder Ejecutivo en 1998 (Honorable Cámara de Senadores, 1998). En este solicitaba al Poder 
Ejecutivo nacional que informara «si se han tomado en cuenta las recomendaciones previstas en la Carta de Venecia del 31 de mayo de 1964, y las pautas establecidas en el Mercosur Turístico», en referencia a diez puntos específicos relacionados con la conservación de las Misiones Jesuíticas Guaraníes. Entre ellos mencionaban: $a$ ) la realización de trabajos arqueológicos y de excavación previos a las obras de restauración teniendo en cuenta la «Recomendación definidora de los principios internacionales que deben ser aplicados en materia de excavaciones arqueológicas» adoptada por la UNESCO en 1956; $b$ ) la realización de estudios de impacto ambiental con relación a las obras; $c$ ) el inicio de acciones conjuntas para asesoramiento técnico nacional e internacional en el marco del Proyecto Misiones del Mercosur Cultural, $d$ ) la adopción de medidas colectivas y acciones conjuntas para la integración del Proyecto Misiones Jesuítico-Guaraníes dentro del Mercosur Cultural; $e$ ) el diseño de circuitos turísticos en el marco del Mercosur Cultural, entre otras.

En la respuesta elaborada por la CNMLyBH se explican las intervenciones que se han realizado en el sitio desde 1940. Asimismo se menciona que en 1996 se elaboró un programa regional para la recuperación y rehabilitación de las Misiones JesuíticoGuaraníes, que contempla la participación de diferentes niveles de gobierno y abarca áreas de obras públicas, turismo, educación, trabajo y desarrollo social. ${ }^{7}$ Este programa fue presentado en el marco del Mercosur Cultural, ${ }^{8}$ que en aquella fecha planteaba como una acción primordial la declaratoria a nivel regional del Itinerario Cultural de las Misiones Jesuíticas Guaraníes, Moxos y Chiquitos como Patrimonio Cultural del Mercosur, la que se concretó en junio de 2015.

En este caso, el Poder Legislativo alegó, para controlar la actuación del Ejecutivo, resoluciones no vinculantes de organizaciones internacionales (recomendaciones) y de organizaciones no gubernamentales (cartas). Estos instrumentos reflejan recomendaciones y reglas que deberían ser aplicadas por los organismos estatales (en este caso la CNMLyBH) a fin de proteger el patrimonio cultural, y su adopción se fundamenta en la constitución experta de los organismos que las dictan. Como tales, son normas de soft law, que en este caso actúan de modo particular. Así, la doctrina asigna dos aspectos característicos a los documentos de soft law: participan orientando la creación de

7 Este programa tiene como antecedente el Plan Director de Acciones para la puesta en valor de las Misiones Jesuíticas que fue elaborado en el marco de un Convenio de Asistencia Técnica con España, entre 1992 y 1995. El Plan Director nunca llegó a implementarse en obras concretas, pero en 1996 fue reformulado como programa de desarrollo social, que incluyó a otras áreas de gobierno y fue presentado en el seno del Mercosur Cultural.

8 Mercosur Cultural es la reunión de ministros de Cultura del Mercosur, creada por decisión 02/95 del CMC (Mercosur, 1995) e institucionalizada a partir de 2014 (decisión 22/14 CMC) con la finalidad de promover la difusión y conocimiento de los valores y tradiciones culturales de los Estados parte y presentar propuestas de coordinación y cooperación en el ámbito cultural (Mercosur, 2014a). Asimismo, desde 2014 se instituyó en este ámbito el Reglamento para el Reconocimiento del Patrimonio Cultural del Mercosur (decisión 21/14 CMC, Mercosur, 2014b). 
normas y actúan como instrumentos interpretativos (Mazuelos Bellido, 2004). Aquí se observa otra función del dispositivo: como instrumento que interfiere en la dialéctica entre el Poder Ejecutivo y el Poder Legislativo. En este caso está respaldada por una solicitud de la Cámara de Senadores de la Nación. Esta función se apoya en cierta auctoritas que posee el instrumento por su elaboración independiente y experta. Ello implica que aquel Estado u organismo que no lo adopte debe justificar este apartamiento. Al decir de Laporta, esta conformidad proviene de factores y causas contextuales que son discutibles y cambiantes (Laporta, 2005, p. 262).

En esta parte se presentaron tres situaciones en las cuales el soft law incidió en la regulación de este sitio del Patrimonio Mundial. En la primera de ellas, el compromiso asumido en una norma jurídicamente no vinculante puso en movimiento la administración pública y generó normas al interior del Estado que permitieron la postulación de las Misiones Jesuíticas Guaraníes a la Lista del Patrimonio Mundial. En el segundo caso, el contenido soft law penetra en el ordenamiento, al servir de argumento para una adecuación de normas subnacionales a estándares provenientes de él. Finalmente, el soft law funciona como guía para la acción de organismos nacionales, y es fundamento de control por parte del Senado.

En los tres casos, la aplicación de normas del soft law internacional tensiona la división de competencias, actualizando los conflictos de competencias entre la nación, las provincias y los municipios.

\section{Reflexiones en torno a la «transmisión del alma» del soft law al ordenamiento jurídico argentino}

Los bienes del Patrimonio Mundial Cultural se encuentran sujetos a un cúmulo de regulaciones de diversos niveles: municipales, provinciales, nacionales e internacionales. En este último nivel se analizó, con especial énfasis, la incidencia del soft law. Es decir, un conjunto de normas emanadas de organismos y organizaciones que no son vinculantes para los Estados nacionales pero que poseen cierta relevancia jurídica. Esta relevancia está dada por su acatamiento por los Estados, en particular por el Poder Ejecutivo, que implementa acciones fundado en estos documentos.

Las razones para este seguimiento por parte de las administraciones estatales son heterogéneas. Estimamos que en el caso estudiado la especificidad de los lineamientos contenidos en estas regulaciones y la experticia de los agentes productores aumentan el valor de estos criterios. A ello se agrega la especialidad de la temática de conservación y tutela del patrimonio cultural, y la ausencia de personal calificado en 
algunas administraciones estatales, con aptitud para generar criterios locales de preservación.

En esta investigación hemos considerado tres tipologías diferenciadas de normas de soft law, que se traducen en los siguientes documentos: la evaluación de ICOMOS, las Directrices Prácticas y las cartas y recomendaciones provenientes de diversas organizaciones y organismos internacionales.

La evaluación de ICOMOS no es un documento vinculante para el Comité del Patrimonio Mundial y tampoco para los Estados parte, es decir que es un documento de soft law. No obstante, en el caso estudiado fue traducida a un instrumento vinculante por el Comité, lo que demuestra el poder que tiene esta organización en este ámbito.

Por una parte, la evaluación es considerada parte de un compromiso político por el cual el Estado nacional debe fomentar un conjunto de acciones y propiciar que los Estados provinciales tomen ciertas decisiones políticas. Estas acciones y decisiones se exteriorizan de diversas maneras y traducen políticas públicas cuyo grado de solidez es variable. Algunas políticas públicas identificadas incluyen, en primer lugar, las acciones encaminadas a elaborar el documento de postulación de los sitios. También, aunque con menor grado de formalización, se explicitan en acciones sobre el territorio.

Por otra parte, resulta destacable que ICOMOS procuró coordinar las acciones de postulación de más de un Estado parte, a partir de considerar los sitios propuestos en categorías patrimoniales que requieren la seriación. En este caso, IComos coordinó las acciones de tutela del patrimonio de los Estados. La organización también lleva adelante esta actividad a partir de estudios temáticos sobre la Lista del Patrimonio Mundial y las Listas Indicativas y estudios técnicos que apoyan la Estrategia global para una Lista del patrimonio mundial equilibrada, representativa y creíble.

La segunda tipología considerada refiere a la aplicación de las Directrices Prácticas. Esta plantea tensiones en el ordenamiento argentino referentes a la competencia de los niveles nacional y subnacionales, por cuanto las postulaciones deben adecuarse a las exigencias establecidas en las Directrices.

Ante la instalación de un complejo industrial cercano a las ruinas de Santa Ana, la operatividad de las Directrices se visibilizó particularmente en acciones de la CNMLyBH. Este organismo nacional operó hacia el nivel internacional, al realizar gestiones ante UNESCO a fin de mostrar que la instalación industrial no afectaba la protección del bien en cuestión. Asimismo, operó hacia el nivel subnacional, al implementar presiones para regular la zona de amortiguación del bien.

Las Directrices operaron, en este caso, como norma de soft law a partir de la proyección de principios y criterios que delinearon la normativa municipal por intermedio de exigencias de la CNMLyBH.

Resulta notable la defensa que encarna la CNMLyBH del entorno de los bienes, cuando conforme la norma jurídica que rige los monumentos y lugares históricos na- 
cionales no se establecía, en ese momento, la tutela de este espacio. ${ }^{9}$ La regulación de la zona de amortiguación en las Directrices Prácticas le permitió a la CNMLyBH actuar en un espacio que tenía vedado conforme a la ley 12.665, ampliando materialmente su competencia y permitiendo su intervención en situaciones que, de otra manera, no hubiese podido considerar.

En tanto, las tensiones generadas en el régimen federal requieren diseñar soluciones específicas. La concertación entre nación, provincias y municipios a través de acuerdos que establezcan pautas de gobierno de los bienes del patrimonio cultural parece ser la posibilidad más cercana, por cuanto ha sido incluida en la reciente reforma de la ley 12.665 (art. 1 ter a), r) y art. 2).

Finalmente, el tercer tipo de instrumento de soft law que se ha implementado en Argentina en relación con los bienes del patrimonio cultural son las cartas y recomendaciones provenientes de UNESCO, OEA, ICOMOS y Mercosur. Estas normas se caracterizan como soft law atento a que su contenido preceptivo no goza de valor obligatorio, aunque contienen normas de conducta que promueven la práctica estatal en cierta dirección. En general se trata de manifestaciones jurídicas de principios y objetivos, y no de reglas detalladas y precisas.

En el caso estudiado el soft law funcionó, a partir de una solicitud de informes, como un instrumento de control de la dialéctica entre el Poder Ejecutivo y el Poder Legislativo. La elaboración de estos documentos por organismos internacionales, con fuerte presencia de expertos, les otorga cierta legitimidad que exige una fundamentación ante el apartamiento de los principios y prácticas que promueven. Esta argumentación, en general, no cuestiona los principios, objetivos o prácticas establecidas en las cartas y recomendaciones, sino que justifica su no implementación en virtud de factores contextuales (carencia de presupuesto, ausencia de técnicos especializados, dificultades en la articulación de diversos niveles de la administración, etc.).

El estudio de caso permite explicitar las modalidades por las cuales opera el soft law en el ámbito interno estatal y categorizarlas a fin de permitir su identificación en otros ámbitos o situaciones. Asimismo, la descripción de las circunstancias de hecho visibiliza la influencia real de estas normas en el derecho nacional y subnacional.

Si la globalización ha conmovido la articulación de ordenamientos jurídicos, la influencia de las normas de soft law representa un nuevo desafío a los operadores jurídicos en al menos dos aspectos. Por una parte, se requieren análisis empíricamente fundados para poner de manifiesto las modalidades de actuación y articulación de estas normatividades. Por otra, resulta necesario construir teorías sofisticadas para resolver las tensiones que se presentan en las estructuras estatales.

9 La ley 12665, que establece la creación de la Comisión Nacional de Monumentos, Bienes y Lugares Históricos fue reformada en 2015, por ley 27103. Incorporó la tutela del entorno de los bienes culturales en el artículo 1 ter g). 


\section{Bibliografía}

AFFolder, N. (2007). «Mining and the world heritage convention: Democratic legitimacy and treaty compliance». Pace Environmental Law Review, 24 (1), 35-66.

ARIÑo Villarroya, A. (2002). «La expansión del patrimonio cultural». Revista de Occidente, $250(\mathrm{~S}), 129-150$.

Bidegain, C. (2001). Curso de derecho constitucional, tomo III. Buenos Aires: AbeledoPerrot.

Blanc Altemir, A. (1992). El patrimonio común de la humanidad: Hacia un régimen jurídico internacional para su gestión. Barcelona: Bosch.

Calvo, J. L. (2018). «Tecnología y nuevo derecho: autorregulación regulada y soft law: A propósito del reglamento europeo de protección de datos». Revista de Privacidad y Derecho Digital, 3(9), 29-68.

CNMLyBH (22 de febrero de 1999). Nota n.o 177 a CONAPLU. Archivo de la CNMLyBH, Buenos Aires.

CNMLyBH (26 de diciembre de 2000). Nota n. 880 al intendente de Santa Ana. Archivo de la CNMLyBH, Buenos Aires.

Comisión TÉcnica del Convenio NACión-Provincia (1998). «Adenda al acta de reunión del mes de diciembre de 1998». En acta de la reunión de la Comisión Técnica del Convenio Nación-Provincia. Archivo de la CNMLyBH, Buenos Aires.

Constitución de la Nación Argentina (1964). Congreso General Constituyente, Buenos Aires, Argentina.

CorvaláN, J. G. (2018). «Administración pública inteligente en Argentina: Un estado de la cuestión». Revista Derecho Administrativo Económico, (25), 7-25.

DEZALAY, Y. y TRUBEK D. (1997). «La reestructuración global y el derecho. La internacionalización de los campos jurídicos y la creación de espacios transnacionales». Pensamiento Jurídico, (1), 5-41.

DulitzKY, A. (2006). «Federalismo y derechos humanos: El caso de la Convención Americana sobre Derechos Humanos y la República Argentina». Anuario Mexicano de Derecho Internacional, (6), 199-249.

Fillol, P. y MárqueZ, J. C. (2018). «Compliance laboral: problemática de la compatibilización de las medidas disciplinarias con la autorregulación de las empresas («Soft Law»): La compatibilidad del derecho laboral con el compliance y los modelos de organización y gestión para la prevención de delitos». Revista Aranzadi Doctrinal, (4), 19-37.

Foy Valencia, P. (2008). «Soft Law y derecho internacional ambiental. Algunas aplicaciones nacionales». Revista Boliviana de Derecho, (5), 65-84. Recuperado de http://www.redalyc.org/articulo.oa?id=427539905005. 
FRIAS, G. (2011). «El proceso federal argentino, su situación actual. Autonomía y dependencia». En A. Hernández y G. BARRERA Buteler (coords.), Derecho público provincial (pp. 121-148). Buenos Aires: Abeledo Perrot.

Galbraith, J. y Zaring, D. (2014). «Soft law as foreign relations law». Cornell Law Review, 99, 735-794. Recuperado de http://cornelllawreview.org/files/2014/05/ 99CLR735.pdf.

GEBERT, D. (1998). «Sovereignty under the World Heritage Convention: A questionable basis for limiting federal land designation pursuant to international agreements». Southern California Interdisciplinary Law Journal, (7S), 427-443.

Gelly y OBes, C. (17 de junio de 1983). Nota al secretario de Cultura de la Nación. Archivo de la CNMLyBH, Buenos Aires.

GonzÁlez Bertomeu, J. (2010). «Notas sobre federalismo». En R. Gargarella (coord.), Teoría y crítica del derecho constitucional (pp. 439-485). Buenos Aires: Abeledo Perrot.

HERNÁNDEZ, A. G. (2018). «Diligencia debida en derechos humanos y empresas transnacionales: De la ley francesa a un instrumento internacional jurídicamente vinculante sobre empresas y derechos humanos». Lex Social: Revista de Derechos Sociales, 8(2), 216-250.

Honorable Cámara de Senadores (3 de junio de 1998). Comunicación n. ${ }^{o} 426 / 98$ al Poder Ejecutivo. Archivo de la CNMLyBH, Buenos Aires.

ICOMOS (s. f.). Carta internacional sobre la conservación y la restauración de monumentos y sitios: Carta de Venecia 1964. Recuperado de https://www.icomos.org/ charters/venice_sp.pdf.

JuRADo, D. M. (2018). «El soft law en derecho administrativo y su control judicial en Colombia». Revista Digital de Derecho Administrativo, 20, 289-243. Recuperado de https://revistas.uexternado.edu.co/index.php/Deradm/article/view/5467/ 6661.

KISS, A. (1982). «La notion de patrimoine commun de l'humanité. Recueil des cours». Académie de Droit International de La Haye, 2(175), 98-256.

LAPORTA, F. (2005). «Globalización e imperio de la ley: Algunas dudas westfalianas». Anales de la Cátedra Francisco Suárez, (39), 243-265.

LEVRAND, N. (2016). «Análisis de la multiplicidad de competencias y regulaciones en un sitio del patrimonio mundial argentino». Inciso, 18(2), 9-19.

LEVRAND, N. (2017). La regulación del patrimonio cultural mundial en Argentina. Dominio, competencias y significaciones. (Tesis doctoral). Universidad Nacional del Litoral: Santa Fe.

Ley n. 12.665 (1940). Creación de la Comisión Nacional de Museos y de Monumentos y Lugares Históricos. Recuperada de http://servicios.infoleg.gob.ar/infolegInternet/ anexos/20000-24999/23121/texact.htm. 
MARTínez, M. (2017). El llamado derecho suave en el derecho de la competencia. (Tesis doctoral). Universidad Complutense de Madrid: Madrid.

Mazuelos Bellido, A. (2004). «Soft Law: ¿Mucho ruido y pocas nueces?». Revista Electrónica de Estudios Internacionales, (8),1-40. Recuperado de www.reei.org/index.php/revista/num8/archivos/MazuelosBellido_reei8_pdf.

Mercosur (1995). Reunión de ministros de Cultura: Decisión - 002/1995. Recuperado de http://gd.mercosur.int/SAM\%5CGestDoc\%5Cpubweb.nsf/6BE911E1D3933D49 03258352006AF128/\$File/DEC_002-1995_ES_ReuMinCult.pdf.

Mercosur (2014a). Estructura orgánica y reglamento interno del Mercosur Cultural: Decisión - 022/2014. Recuperado de http://gd.mercosur.int/SAM\%5CGestDoc\% 5Cpubweb.nsf/34CBA1E0D4DD4CF803258352006B7E59/\$File/DEC_022-2014_ ES_Estruct\%20y\%20Reg\%20Int\%20MCS\%20Cultural.pdf.

Mercosur (2014b). Patrimonio cultural del Mercosur: Decisión 021/2014. Recuperado de http://gd.mercosur.int/SAM\%5CGestDoc\%5Cpubweb.nsf/190E428D919E 842B03258352006BD0AB/\$File/DEC_021-2014_ES_Patrimonio\%20Cultural\% 20MCS.pdf.

MuRIEL, D. (2010). «El patrimonio como dispositivo de construcción de lo nuestro en tiempos de... ¿crisis? De la herencia cultural a las identidades: nuevas formas de hacer comunidad en la contemporaneidad». En P. DE MARINIS, G. GATTI CASAL DE Rey e I. IRAZUZTA Di ChiARA (eds.), La comunidad como pretexto: en torno al (re)surgimiento de las solidaridades comunitarias (pp. 307-344). Barcelona: Anthropos.

Normas de Quito: Informe final de la reunión sobre conservación y utilización de monumentos y lugares de interés histórico y artístico (1967). Recuperado de http://www.planmaestro.ohc.cu/recursos/papel/cartas/1967-quito.pdf.

Nota WXC/74/201/041/HvH/cc (4 de febrero de 1999). Archivo de la CNMLyBH, Buenos Aires.

OnETTO, C. (1999). San Ignacio Miní: Un testimonio que debe perdurar. Buenos Aires: Dirección Nacional de Arquitectura.

RATneR, S. (1998). «International law: The trials of global norms». Foreign Policy, (110), 65-80. Recuperado de https://www.jstor.org/stable/1149277?seq=1\#page_ scan_tab_contents.

Schávelzon, D. (2008). Mejor olvidar. Buenos Aires: De los Cuatro Vientos.

Secretaría de Cultura (2000). Expediente 868/00. Archivo de la CNMLyBH, Buenos Aires.

Silva, F. (1996). As ciudades brasileiras e o patrimonio cultural da humanidade. San Pablo: Peirópolis.

TERPAN F. (2015). «Soft Law in the European Union: The changing nature of EU law». European Law Journal, 21 (1), 68-96. 
UNESCO (1963). «Recomendación relativa a la Protección de la Belleza y el Carácter de los Lugares y Paisajes». En Actas de la conferencia general: 12. ${ }^{a}$ reunión, París, 1962. Resoluciones (pp. 143-146). París: UNESCO. Recuperado de http://unesdoc. unesco.org/images/0011/001145/114582s.pdf\#page=146.

UNESCO (1973). «Convención sobre la Protección del Patrimonio Mundial Cultural y Natural». En Actas de la Conferencia General: 17. reunión, París, 17 de octubre 21 de noviembre de 1972. Vol. 1. Recomendaciones (pp. 140-151). París: UNESCO. Recuperado de http://portal.unesco.org/es/ev.php-URL_ID=13055\&URL_DO= DO_TOPIC\&URL_SECTION=201.html.

UNESCO (1983). «Convention concerning the protection of the world cultural and natural heritage». En World Heritage Committee, $7^{\text {th }}$ ordinary session, Florence, Italy, 5-9 December 1983: Report of the rapporteur. Recuperado de http://whc. unesco.org/archive/1983/sc-83-conf009-8e.pdf.

UNESCO (1984). «Convention concerning the protection of the world cultural and natural heritage». En World Heritage Committee, $8^{\text {th }}$ ordinary session, Buenos Aires, Argentina, 29 October-2 November 1984: Report of the rapporteur. Recuperado de http://whc.unesco.org/archive/1984/sc-84-conf004-9e.pdf.

UNESCO (1997). Convention concernant la protection du patrimoine mondial, culturel et naturel: Rapport. París: UNESCO. Recuperado de http://whc.unesco.org/archive/ 1996/whc-96-conf201-21f.pdf.

UNESCO (2008). Directrices Prácticas para la aplicación de la Convención del Patrimonio Mundial. Recuperado de http://whc.unesco.org/archive/opguide08-es.pdf.

ZACHARIAS, D. (2010). «The UNESCO regime for the protection of World Heritage as prototype of an autonomy-gaining international institution». En: A. VON BoGDANDY et al. (eds.) The exercise of public authority by international institutions, (pp. 301336.). Heidelberg: Springer. 